\title{
Classification of EUV stellar sources detected by the ROSAT $\mathrm{WFC}^{\star, \star \star}$
}

\author{
I. Photometric and radial velocity studies

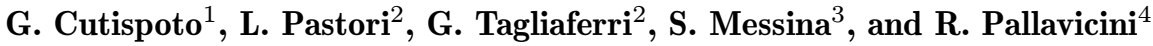 \\ 1 Catania Astrophysical Observatory, V.le A. Doria 6, I-95125 Catania, Italy \\ 2 Brera Astronomical Observatory, Via Bianchi 46, I-22055 Merate, Italy \\ 3 Institute of Astronomy, Catania University, V.le A. Doria 6, I-95125 Catania, Italy \\ 4 Palermo Astronomical Observatory, P.zza del Parlamento 1, I-90134 Palermo, Italy
}

Received March 2; accepted May 19, 1999

\begin{abstract}
We present the results of high-precision $U B V(R I)_{\text {c }}$ photometric observations and of spectroscopic radial velocity measurements obtained at the European Southern Observatory for a sample of 51 cool stars detected in the EUV by the ROSAT Wide Field Camera (WFC). Using also recent results from HIPPARCOS, we infer spectral types and investigate the single or binary nature of the sample stars. Optical variability, with periods in the $0.4-13$ day range, has been detected for the first time in 15 of these stars.
\end{abstract}

Key words: stars: activity — stars: late-type — stars: pre-main sequence - stars: variables - X-rays: stars

\section{Introduction}

Large samples of stellar X-ray sources have been discovered serendipitously by the Einstein, EXOSAT and ROSAT Observatories. Optical follow-up studies have demonstrated that a large fraction of these samples is composed by active binaries, by pre-main sequence and other very young stars and by flare stars (see, among others, Fleming et al. 1988, 1989a, 1989b; Favata et al. 1993, 1995; Jeffries et al. 1995; Neuhäuser et al. 1997).

Send offprint requests to:

G. Cutispoto (gcutispoto@alpha4.ct.astro.it)

* based on data collected at the European Southern Observatory, La Silla, Chile.

** Tables 1-5, Figs. 2-27 and the complete data set are available in electronic form at the CDS via anonymous ftp to cdsarc.u-strasbg.fr or via http://cdsweb.ustrasbg.fr/Abstract.html
Over the past few years our group has carried out extensive photometric and spectroscopic observations of cool stars serendipitously detected by EXOSAT (Tagliaferri et al. 1992, 1994; Cutispoto et al. 1996). We have shown that, in addition to dMe flare stars, at least one third of the EXOSAT serendipitous sources is constituted by young stars, with ages comparable to or younger than the Pleiades. Another third consists of RS CVn binaries, while the physical nature of the remaining sources is more uncertain: they could be either young objects or very active binaries. A large fraction of these stars is variable at optical wavelengths, with the observed variability best interpreted as produced by photospheric cool spots. Similar results have been obtained for the samples of cool stars selected with the Einstein satellite (Fleming et al. 1988, 1989a, 1989b; Favata et al. 1993, 1995).

Probably, the most interesting result of these surveys is that there seems to be an excess of young stars, near ZAMS or even younger, with respect to what is predicted by Galaxy models. This result is now confirmed by other authors using ROSAT data. For instance, Jeffries (1995) determined the $\mathrm{Li}$ abundances for a sample of late-type stars EUV-selected with the WFC on board ROSAT. He confirmed that a high portion of these stars are as young as or younger than the Pleiades and, based on their kinematics, he suggested that these stars are part of a group of young open clusters and nearby B stars known as the Local Association. Finally, Neuhäuser et al. (1997) performed an optical follow-up on 111 late-type stars detected in the RASS south of the Taurus molecular cloud. Among these off-cloud stars 24 have a Li excess and were classified either as PMS (9) or as young ZAMS (15) objects.

Following our works on the EXOSAT sample, we defined a new sample of active cool stars EUV-selected with the ROSAT WFC (Pounds et al. 1993; Pye et al. 1995) and 
performed spectroscopic (Li I $6708 \AA$ A $\mathrm{H} \alpha$ and Ca II H\&K lines) and photometric observations, using the CAT and $50 \mathrm{~cm}$ ESO telescopes in the South, the McMath telescope at Kitt Peak and the $80 \mathrm{~cm}$ APT telescope at Catania Observatory in the North.

In this paper we present a subset of these data, that include photometric observations and RV measurements for the stars in our sample that are observable from the southern hemisphere.

\section{Data sample and observations}

\subsection{The sample}

In order to test the findings on previous samples of $\mathrm{X}$-ray selected stars, we selected a new large sample of active nearby late type stars. To this end we used the data from the EUV all-sky survey obtained with the Wide Field Camera (WFC) on board the ROSAT satellite. The WFC survey is dominated by active late type stars, that constitute more than one third of the full sample (Pounds et al. 1993; Pye et al. 1995). Given that the absorption by the interstellar medium is very efficient at EUV wavelengths, the WFC survey is mainly made of stars in the solar neighbourhood and/or very active. Thus, it is an ideal database to start with.

From the 479 sources listed in the 2RE WFC catalogue (Pye et al. 1995) we selected 190 stars of spectral type between $\mathrm{F}$ and $\mathrm{K}$ and with magnitude mainly in the $5<V<10$ range. We concentrated on this range of magnitudes for two reasons. On the one side stars brighter than $5^{\text {th }}$ magnitude are usually well studied and the relevant information on them can be found in the literature. On the other side stars weaker than $10^{\text {th }}$ magnitude are too faint to be studied with the telescopes we used, in particular with respect to the high resolution spectroscopic observations. Nevertheless, we left in our sample few brighter (one in the sub-sample presented here) and weaker (two in this sub-sample) stars. Moreover, we used the information found in the literature, in particular using the SIMBAD database, to exclude all stars that are already well known and for which high resolution spectroscopy is already available (e.g. many of the most known and active RS CVn binaries that are in the WFC catalogue). At the end we obtained a list of 104 stars for which, when we started this project, we could not find detailed information in the literature with respect to at least one of the spectral lines we were interested in, i.e. $\mathrm{Li} 6708 \AA, \mathrm{H} \alpha$ and $\mathrm{Ca}$ II H\&K lines. Among the selected stars, HD 71285 is present in Pounds et al. (1993) WFC-BSC catalogue but not in Pye et al. (1995) WFC-2RE catalogue. In fact the detection of HD 71285, although real, is just below the acceptance criteria for the 2RE. Finally, we note that 13 stars (i.e. HD 16699, HD 35114, HD 36869, HD 124672, HD 143937, HD 156498,
HD 195434, SAO 91772, SAO 111210, SAO 150508, SAO 150676, SAO 196024, SAO 234124) are listed in the $2 \mathrm{RE}$ but not in the BSC catalogue, due to an improved detection algorithm (see Pye et al. 1995). Among the 104 stars of our list 51 were suitable for observations in the southern hemisphere and were indeed observed with the $1.4 \mathrm{~m}$ ESO CAT telescope in various observing runs. Photometric observations of 45 stars were obtained with the $50 \mathrm{~cm}$ ESO telescope, while for the remaining six stars of the sample we used the information obtained from either the HIPPARCOS output star catalogue or the literature.

\subsection{Photometric observations}

For all stars but six $U B V(R I)_{\mathrm{c}}$ multicolor photometry has been obtained and has been used to determine the physical parameters and the distance of the stars (see Sect. 3.4). In particular, the data presented in Table 1 were collected over the intervals 19 November - 03 December 1993 (N93), 24 November - 04 December 1994 (N94), 15-28 January 1995 (J95), 19 August - 03 September 1995 (A95) and 03-30 December 1996 (D96) at the European Southern Observatory (La Silla, Chile) by using the $50 \mathrm{~cm}$ ESO telescope equipped with a single channel photon-counting photometer, a thermoelectrically cooled Hamamatzu R943/02 photomultiplier and standard ESO filters matching the $U B V(R I)_{\mathrm{c}}$ system. Table 1 also includes data for the two short period eclipsing binaries HD 9770 and HD 195434, already published by Cutispoto et al. (1997a) and those of a preliminary study of some of the stars in our sample presented by Cutispoto et al. (1995). Finally, as our list includes a few well-known active stars, we could also use magnitude and colors obtained with the same instrument by our group and already available in the literature. The typical accuracy of our absolute photometry, details are given in Cutispoto (1998), is of the order of 0.01 magnitudes. For the six stars we were unable to observe, the $V$ data are from the HIPPARCOS output star catalogue or from the literature; their visual magnitudes are marked with "*" in Table 1 . Information on optical variability, that was detected for the first time in 15 stars of our sample, and is likely to be due to the presence of photospheric spots, is given in Table 2. There, together with the brightest magnitude $\left(V_{\mathrm{m}}\right)$ and the light curve amplitude observed by us, we also give the brightest visual magnitude $\left(V_{\min }\right)$ reported in the literature, in order to ascertain the presence of long-term variability. In the case of eclipsing binaries, the amplitude of the light curve refers to the out-of-eclipse data. A preliminary version of the photometric section of this paper was given by Cutispoto et al. (1998). The results presented here are based on a refined analysis of our photometric data. 
Table 1. Star name, maximum luminosity and colors, epoch of observations, spectral type inferred from the colors, parallax measured by the HIPPARCOS satellite, photometric parallax

\begin{tabular}{|c|c|c|c|c|c|c|c|c|c|}
\hline Name & $V_{\mathrm{m}}$ & $U-B$ & $B-V$ & $V-R$ & $V-I$ & Epoch & Spectral Type & $d(\mathrm{H})$ & $d(\mathrm{ph})$ \\
\hline HD 2133 & 9.59 & 0.07 & 0.57 & 0.32 & 0.61 & A95 & F9V/IV + WD & 140 & 142 \\
\hline HD 6628 & 7.70 & 0.36 & 0.87 & 0.50 & 1.00 & N93 & $\mathrm{K} 1 / 2 \mathrm{IV}+\mathrm{F} 8 \mathrm{~V}$ & 132 & 134 \\
\hline HD 8357 & 7.24 & 0.38 & 0.85 & 0.50 & 1.00 & P1 & $\mathrm{K} 1 \mathrm{IV} / \mathrm{V}+\mathrm{G} 5 / 6 \mathrm{~V}$ & 45 & 46 \\
\hline HD 8358 & 8.08 & 0.13 & 0.70 & 0.41 & 0.86 & P2 & G5V + G5V & 66 & 60 \\
\hline HD 9770 & 7.11 & 0.58 & 0.92 & 0.54 & 1.07 & N94 & $\mathrm{K} 0 / 1 \mathrm{~V}+\mathrm{K} 3 \mathrm{~V}+\mathrm{K} 3 / 4 \mathrm{~V}$ & 24 & 23 \\
\hline HD 16699 & 7.34 & 0.10 & 0.61 & 0.36 & 0.68 & A95 & $\mathrm{F} 9 \mathrm{~V}+\mathrm{K} 0: \mathrm{V}$ & 61 & 63 \\
\hline HD 18131 & 7.32 & 0.74 & 0.98 & 0.52 & 0.97 & N93 & $\mathrm{K} 1 \mathrm{IV}+\mathrm{WD}$ & 105 & 96 \\
\hline HD 24916 & 8.03 & 1.03 & 1.13 & 0.68 & 1.30 & N94 & $\mathrm{K} 4 / 5 \mathrm{~V}+\mathrm{M} 2 \mathrm{Ve}$ & 16 & 15 \\
\hline HD 25457 & $5.34^{*}$ & 0.00 & 0.52 & 0.30 & 0.58 & & $\mathrm{~F} 6 / 7 \mathrm{~V}$ & 19 & 22 \\
\hline HD 32008 & $5.37^{*}$ & 0.34 & 0.80 & & & & G7IV/III + WD & 55 & 51 \\
\hline HD 33262 & $4.68^{*}$ & -.03 & 0.52 & 0.31 & 0.60 & & F7V & 12 & 16 \\
\hline HD 35114 & 7.34 & -.02 & 0.49 & 0.29 & 0.56 & A95 & $\mathrm{F} 5 / 6 \mathrm{~V}$ & 46 & 60 \\
\hline HD 36869 & 8.46 & 0.13 & 0.64 & 0.37 & 0.70 & J95 & G3V & 35: & 57 \\
\hline HD 37572 & 7.86 & 0.41 & 0.82 & 0.47 & 0.88 & J95 & $\mathrm{K} 0 \mathrm{~V}$ & 24 & 25 \\
\hline HD 41824 & 6.62 & 0.26 & 0.72 & 0.41 & 0.78 & J95 & $\mathrm{G} 5 \mathrm{~V}+\mathrm{K} 7: \mathrm{V}+\mathrm{G} 6 \mathrm{~V}$ & 30 & 28 \\
\hline HD 43162 & 6.37 & 0.22 & 0.70 & 0.39 & 0.75 & J95 & $\mathrm{G} 5 / 6 \mathrm{~V}$ & 17 & 18 \\
\hline HD 43989 & 7.91 & 0.05 & 0.57 & 0.33 & 0.64 & N93 & F9V & 50 & 59 \\
\hline HD 45081 & 9.80 & 0.90 & 1.13 & 0.68 & 1.32 & J95 & K5 PMS(?) & 38 & $>33$ \\
\hline HD 48189 & 6.14 & 0.07 & 0.61 & 0.36 & 0.70 & J95 & $\mathrm{G} 0 \mathrm{~V}+\mathrm{K} 2 / 3 \mathrm{~V}$ & 22 & 26 \\
\hline HD 71285 & 7.83 & 0.12 & 0.61 & 0.34 & 0.67 & J95 & $\mathrm{G} 1 \mathrm{~V}+\mathrm{G} 3 \mathrm{~V}$ & 66 & 67 \\
\hline HD 75997 & 9.04 & 0.37 & 0.85 & 0.51 & 1.02 & D96 & $\mathrm{K} 0 / 1 \mathrm{~V}$ & 31: & 39 \\
\hline HD 78644 & 8.27 & 0.13 & 0.65 & 0.38 & 0.73 & J95 & $\mathrm{G} 3 \mathrm{~V}+\mathrm{M} 0: \mathrm{V}$ & 52 & 51 \\
\hline HD 82159 & $8.66^{*}$ & & 0.91: & & 0.91: & & $\mathrm{G} 9 \mathrm{~V}+\mathrm{K} 4: \mathrm{V}$ & 47: & 44 \\
\hline HD 82558 & 7.80 & 0.57 & 0.90 & 0.53 & 1.02 & $\mathrm{P} 4$ & $\mathrm{~K} 2 \mathrm{~V}$ & 18 & 19 \\
\hline HD 96064 & 7.57 & 0.34 & 0.76 & 0.41 & 0.80 & J95 & G8V & 25 & 26 \\
\hline HD 118100 & $9.24^{*}$ & 1.04 & 1.21 & 0.73 & 1.40 & & $\mathrm{~K} 6: \mathrm{V}$ & 18 & 18 \\
\hline HD 124672 & 7.54 & -.04 & 0.49 & 0.29 & 0.58 & A95 & $\mathrm{F} 5 \mathrm{~V}+\mathrm{K} 4: \mathrm{V}$ & 72 & 70 \\
\hline HD 140637 & 9.21 & 0.68 & 1.02 & 0.60 & 1.21 & J95 & K3 PMS & 41 & $>31$ \\
\hline HD 141943 & 7.87 & 0.10 & 0.63 & 0.35 & 0.69 & J95 & G2 PMS & $>50$ & $>46$ \\
\hline HD 143937 & 8.61 & 0.41 & 0.85 & 0.51 & 0.98 & A95 & $\mathrm{G} 9 \mathrm{~V}+\mathrm{M} 0: \mathrm{V}+\mathrm{K} 7: \mathrm{V}$ & 42 & 41 \\
\hline HD 156498 & 8.45 & 0.19 & 0.70 & 0.44 & 0.84 & A95 & $\mathrm{G} 4 \mathrm{~V} / \mathrm{IV}+\mathrm{M} 0: \mathrm{V}+\mathrm{G} 8: \mathrm{V}$ & 83 & 84 \\
\hline HD 171488 & 7.44 & 0.09 & 0.63 & 0.36 & 0.70 & A95 & $\mathrm{G} 2 \mathrm{~V}$ & 37 & 37 \\
\hline HD 195434 & 8.71 & 0.47 & 0.87 & 0.53 & 1.01 & A95 & $\mathrm{K} 2 \mathrm{~V}+\mathrm{K} 6 \mathrm{~V}+\mathrm{K} 2 \mathrm{~V}$ & 44 & 44 \\
\hline HD 197890 & 9.32 & 0.54 & 0.93 & 0.56 & 1.11 & $\mathrm{P} 5$ & K2/3 PMS & 44 & $>36$ \\
\hline HD 199143 & 7.26 & 0.03 & 0.53 & 0.31 & 0.62 & A95 & F7V & 48 & 51 \\
\hline HD 217411 & 9.57 & 0.17 & 0.66 & 0.37 & 0.74 & A95 & $\mathrm{G} 4 / 5 \mathrm{~V}+\mathrm{WD}$ & $>48$ & 87 \\
\hline HD 222259 & 8.18 & 0.27 & 0.76 & 0.44 & 0.86 & N93 & $\mathrm{G} 5 / 6 \mathrm{~V}+\mathrm{K} 4: \mathrm{V}$ & 46 & 44 \\
\hline HD 223816 & 9.84 & -.09 & 0.49 & 0.28 & 0.54 & A95 & F5V + WD & $>33$ & 200 \\
\hline HD 291095 & 8.98 & 0.57 & 0.93 & 0.52 & 1.02 & N93 & $\mathrm{K} 3 \mathrm{~V} / \mathrm{IV}+\mathrm{G} 6 \mathrm{~V}$ & 43: & 102 \\
\hline BD-00 1712 & 9.34 & 0.78 & 1.03 & 0.64 & 1.23 & J95 & $\mathrm{K} 3 \mathrm{~V}+\mathrm{M} 0: \mathrm{V}$ & $>24$ & 36 \\
\hline $\mathrm{BD}+08102$ & 10.04 & 0.37 & 0.86 & 0.48 & 0.97 & D96 & $\mathrm{K} 2 \mathrm{~V}+\mathrm{WD}$ & $>15$ & 54 \\
\hline $\mathrm{BD}+0973$ & 10.43 & 0.92 & 1.16 & 0.73 & 1.49 & D96 & $\mathrm{K} 5 / 6: \mathrm{V}+\mathrm{M} 4: \mathrm{V}+\mathrm{WD}$ & 33 & 38: \\
\hline SAO 91772 & 8.44 & 0.26 & 0.76 & 0.46 & 0.93 & A95 & $\mathrm{G} 7 / 8 \mathrm{~V}+\mathrm{M} 0: \mathrm{V}$ & 41 & 40 \\
\hline SAO 111210 & 9.49 & 0.25 & 0.76 & 0.45 & 0.88 & D96 & $\mathrm{G} 6 \mathrm{~V}+\mathrm{K} 3 \mathrm{~V}$ & $>37$ & 79 \\
\hline SAO 150508 & 9.62 & 0.17 & 0.69 & 0.40 & 0.77 & D96 & $\mathrm{G} 5 / 6 \mathrm{~V}$ & 24: & 80 \\
\hline SAO 150676 & 8.97 & 0.08 & 0.62 & 0.36 & 0.70 & D96 & G2V (PMS ?) & $>70$ & 76 \\
\hline SAO 151224 & 9.26 & 0.67 & 1.04 & 0.61 & 1.17 & N93 & $\mathrm{K} 3 \mathrm{~V} / \mathrm{IV}+\mathrm{K} 1 \mathrm{~V} / \mathrm{IV}$ & $>59$ & 176 \\
\hline SAO 196024 & 9.52 & 0.38 & 0.83 & 0.49 & 0.96 & D96 & $\mathrm{K} 0 \mathrm{~V}$ & $>37$ & 53 \\
\hline SAO 234124 & 9.35 & 0.44 & 0.86 & 0.49 & 0.99 & D96 & $\mathrm{K} 0 \mathrm{~V}+\mathrm{M} 0 \mathrm{~V}$ & 50 & 51 \\
\hline SAO 243278 & $8.02 *$ & 0.27 & 0.73 & & & & $\mathrm{G} 6 \mathrm{~V}+\mathrm{K} 0: \mathrm{V}$ & 43 & 45 \\
\hline
\end{tabular}

Epoch: (N93) = 19 Nov. - 03 Dec. 1993; (N94) = 24 Nov. - 04 Dec. 1994; (J95) = 15-28 Jan. 1995; (A95) = 19 Aug. - 03 Sep. 1995; $($ D96 $)=03-30$ Dec. 1996; $(\mathrm{P} 1)=$ A\&AS 89, 435; (P2) = A\&AS 84, 397; (P3) = A\&AS 115, 41; (P4) = A\&AS 131, 321; $(\mathrm{P} 5)=$ IBVS 4419. 
Table 2. Star name, variable name, maximum luminosity reported in the literature $\left(V_{\min }\right)$, maximum luminosity $\left(V_{\mathrm{m}}\right)$ and amplitude of variation $(\Delta V)$ observed by us, photometric or orbital period measured by us or from the literature (the latter are

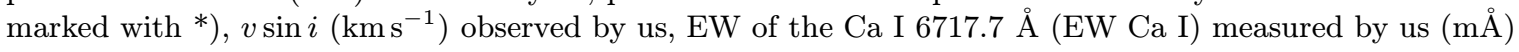

\begin{tabular}{|c|c|c|c|c|c|c|c|}
\hline Star name & Variable name & $V_{\min }$ & $V_{\mathrm{m}}$ & $\Delta V$ & Period & $v \sin i$ & EW Ca I \\
\hline HD 2133 & & 9.61 & 9.59 & 0.03 & & $24 \pm 3$ & $132^{e}$ \\
\hline HD 6628 & CS Cet & 7.68 & 7.70 & 0.05 & $>13$ & $11 \pm 2$ & 160 \\
\hline HD 8357 & AR Psc & 7.24 & 7.24 & 0.08 & 12.245 & $8 \pm 2$ & 158 \\
\hline HD 8358 & BI Cet & 8.08 & 8.20 & 0.09 & $0.515503^{*}$ & $>60^{a}$ & $74+77$ \\
\hline HD $9770^{b}$ & $\mathrm{BB} \mathrm{Scl}$ & 7.09 & 7.11 & 0.04 & 0.4765318 & $<5$ & var \\
\hline HD 16699 & & $7.32^{c}$ & $7.34^{c}$ & 0.02 & & $11 \pm 3$ & 133 \\
\hline HD 18131 & & 7.32 & 7.32 & 0.01 & & $<5$ & 200 \\
\hline HD $24916^{b}$ & & 8.00 & 8.03 & 0.01 & & $9 \pm 2$ & 270 \\
\hline HD 25457 & & 5.34 & & & & $17 \pm 2$ & $122^{e}$ \\
\hline HD 32008 & & 5.37 & & & & $5 \pm 2$ & 132 \\
\hline HD 33262 & & 4.68 & & & & $14 \pm 2$ & $103^{e}$ \\
\hline HD 35114 & & 7.39 & 7.34 & 0.04 & 0.690 & $>60$ & $135^{e}$ \\
\hline HD 36869 & & 8.46 & 8.46 & 0.04 & 1.31 & $28 \pm 2$ & $170^{e}$ \\
\hline HD 37572 & UY Pic & 7.91 & 7.86 & 0.06 & 4.52 & $9 \pm 2$ & $191^{e}$ \\
\hline HD $41824^{b}$ & & 6.56 & 6.62 & 0.04 & 3.3 & $8 \pm 2$ & 161 \\
\hline HD 43162 & & 6.37 & 6.37 & 0.03 & 7.2 & $6 \pm 2$ & $145^{e}$ \\
\hline HD 43989 & V1538 Ori & 7.95 & 7.91 & 0.08 & 1.15 & $47 \pm 3$ & $123^{e}$ \\
\hline HD 45081 & AO Men & 9.84 & 9.80 & 0.09 & 2.65 & $17 \pm 2$ & $280^{e}$ \\
\hline HD $48189^{b}$ & & 6.15 & 6.14 & 0.05 & 2.60 & $14 \pm 2$ & 125 \\
\hline HD 71285 & & 7.86 & 7.83 & 0.04 & 1.35 & $31 \pm 2$ & 150 \\
\hline HD 75997 & & 8.95 & 9.04 & 0.12 & 0.4085 & $>60$ & \\
\hline HD 78644 & & 8.22 & 8.27 & 0.09 & 0.840 & $59 \pm 8$ & 170 \\
\hline HD 82159 & & 8.66 & & & & $13 \pm 2$ & 208 \\
\hline HD 82558 & LQ Hya & 7.77 & 7.80 & 0.12 & 1.61 & $26 \pm 2$ & $237^{e}$ \\
\hline HD 96064 & & 7.57 & 7.57 & 0.04 & 6.9 & $7 \pm 2$ & $165^{e}$ \\
\hline HD 118100 & EQ Vir & 9.24 & & & & $13 \pm 2$ & $315^{e}$ \\
\hline HD 124672 & & 7.54 & 7.54 & 0.08 & 0.646 & $>60$ & 109 \\
\hline HD 140637 & KW Lup & 9.27 & 9.21 & 0.11 & 2.72 & $14 \pm 2$ & $236^{e}$ \\
\hline HD 141943 & & 7.87 & 7.87 & 0.04 & 2.20 & $37 \pm 2$ & $152^{e}$ \\
\hline HD $143937^{b}$ & V1044 Sco & 8.58 & 8.61 & 0.06 & 0.91 & $42 \pm 2$ & $150+90$ \\
\hline HD $156498^{b}$ & V2369 Oph & 8.45 & 8.45 & 0.07 & 0.655 & $>60$ & 90 \\
\hline HD 171488 & V889 Her & 7.39 & 7.44 & 0.04 & $1.338^{*}$ & $40 \pm 2$ & $162^{e}$ \\
\hline HD $195434^{d}$ & MR Del & 8.72 & 8.71 & 0.05 & 0.52175 & $8 \pm 2$ & 88 \\
\hline HD 197890 & BO Mic & 9.34 & 9.32 & 0.10 & 0.380 & $>60$ & 355 \\
\hline HD 199143 & & 7.24 & 7.26 & 0.03 & & $>60$ & $138^{e}$ \\
\hline HD 217411 & & 9.72 & 9.57 & 0.02 & & $6 \pm 2$ & 109 \\
\hline HD $222259^{b}$ & DS Tuc & 8.17 & 8.18 & 0.03 & 1.54 & $17 \pm 2$ & 158 \\
\hline HD 223816 & & 9.88 & 9.84 & 0.02 & & $29 \pm 2$ & $114^{e}$ \\
\hline HD 291095 & V1355 Ori & 8.91 & 8.98 & 0.37 & 3.82 & $47 \pm 7$ & 225 \\
\hline BD-00 $1712^{b}$ & & 9.34 & 9.34 & 0.04 & $1.412^{*}$ & $25 \pm 2$ & $172+132$ \\
\hline $\mathrm{BD}+08102$ & BL Psc & 10.05 & 10.04 & 0.09 & 0.2937 & $>60$ & 299 \\
\hline BD+09 73 & BK Psc & 10.41 & 10.43 & 0.06 & 2.24 & $18 \pm 2$ & 283 \\
\hline SAO 91772 & LN Peg & 8.40 & 8.44 & 0.07 & 1.820 & $26 \pm 2$ & 184 \\
\hline SAO $111210^{b}$ & & 9.56 & 9.49 & 0.07 & 0.5473 & $52 \pm 2$ & 124 \\
\hline SAO 150508 & & 9.59 & 9.62 & 0.05 & 1.90 & $20 \pm 2$ & $160^{e}$ \\
\hline SAO 150676 & & 8.97 & 8.97 & 0.05 & 1.78 & $28 \pm 2$ & $146^{e}$ \\
\hline $\mathrm{SAO} 151224^{b}$ & HY CMa & 9.14 & 9.26 & 0.27 & 4.997 & $39 \pm 4$ & $250+90$ \\
\hline SAO 196024 & & 9.63 & 9.52 & 0.09 & 1.422 & $18 \pm 2$ & $196^{e}$ \\
\hline SAO 234124 & XZ Pic & 9.30 & 9.35 & 0.11 & 1.87 & $23 \pm 2$ & 214 \\
\hline SAO $243278^{b}$ & & 8.02 & & & $0.82376^{*}$ & $44 \pm 2$ & $130+100$ \\
\hline
\end{tabular}

Notes: (a) both components have $v \sin i>60$; (b) the photometry refers to the whole system, the $v \sin i$ only to the primary component; (c) SAO 232842 is included; (d) the photometry refers to the whole system, the $v \sin i$ only to the secondary component; (e) used for the Ca I $6717.7 \AA$ vs. $B-V$ calibration. 


\subsection{Spectroscopic observations}

High resolution spectroscopy has been obtained at ESO (La Silla, Chile) during two different runs, 20-27 January and 24-30 August 1995, using the Coudé Echelle Spectrometer (CES) fed by the $1.4 \mathrm{~m}$ CAT Telescope. Coupling the CES with the Long Camera and a CCD detector, the set-up was fixed to a nominal resolving power of about $60000(\mathrm{H} \alpha)$ and $65000(\mathrm{Li}, \mathrm{Ca})$ for the January run and of about $110000(\mathrm{H} \alpha)$ and 120000 (Li, Ca) for the August run. In January we used a $1024 \times 640$ pixel CCD, resulting in a scale 0.1 (red) and 0.06 (blue) $\AA /$ pixel. In August a $2048 \times 2048$ pixel CCD was employed, resulting in a scale of 0.07 (red) and 0.04 (blue) $\AA$ /pixel. Almost all integrations have a typical signal-to-noise ratio greater than $150-200$ and were made primarily in three wavelength regions centered at $6705 \AA, 6560 \AA$, and $3935 \AA$ (see Table 3). The data reduction was performed by using the IRAF package. After bias subtraction, the spectra were flat-fielded by using the spectrum of a quartz lamp. A Th-Ar lamp was used for wavelength calibration.

\section{Results}

\subsection{Optical variability and period determination}

For the stars showing optical variability, we have determined their photometric periods by fitting sine waves to the $V$-band data and searching for the minimum $\chi^{2}$. See Cutispoto et al. (1995) for further details. In fact, one of the principal results of this study is the discovery of the photometric variability of the following 15 stars:

HD 35114, HD 36869, HD 41824, HD 43162, HD 48189, HD 71285, HD 75997, HD 78644, HD 96064, HD 124672, HD 141943, SAO 111210, SAO 150508, SAO 150676, SAO 196024.

\subsection{Radial velocities}

The properties inferred from the strength and characteristics of the Li I $(6708 \AA), H \alpha(6563 \AA)$ and Ca II K (3933 $\AA$ ) lines are discussed in full detail in Tagliaferri et al. (1999). Here we present the results of radial velocity (RV) determinations, that we use to ascertain the single or binary nature of the stars in our sample. The $6705 \AA$ region contains up to about 20 unblended lines of various strengths suitable for accurate radial velocities measurements as well as the $3935 \AA$ region. The $6560 \AA$ region is half-filled by the $\mathrm{H} \alpha$ line but moderately strong, unblended metallic lines suitable for RV measurements are still available. The spectral regions, resolution and the wavelength of the lines are given in Tables 3 and 4 . The individual lines used for the RV computation are listed in Table 4 . The RV data reduction was performed by standard procedures within the
Table 3. Spectral region and resolution

\begin{tabular}{lcl}
\hline 20-27 January 1995 & & \\
Spectral region $(\AA)$ & Resolution & Notes \\
\hline $6680-6730$ & 65000 & Li region \\
$6530-6590$ & 60000 & H $\alpha$ region \\
$3920-3950$ & 65000 & Ca II region \\
\hline \hline $24-30$ August 1995 & & \\
Spectral region $(\AA)$ & Resolution & Notes \\
\hline $6670-6745$ & 122000 & Li region \\
$6525-6600$ & 110000 & H $\alpha$ region \\
$3915-3960$ & 122000 & Ca II region \\
\hline
\end{tabular}

IRAF package, fitting, by Gaussian profiles, the strongest lines present in each spectrum. The resulting RVs from the individual lines have been averaged and heliocentric correction has been applied. The typical RV accuracy for sharp-lined stars for each line is of the order of $\pm 1 \mathrm{~km} \mathrm{~s}^{-1}$. The final results are listed in Table 5 .

\subsection{Rotational velocities}

We also computed the $v \sin i$ values from the spectra in the $6705 \AA$ region by using the cross correlation task "FXCORR" of the IRAF package. The FWHM of the lines thus obtained can be used to estimate the $v \sin i$ (Soderblom et al. 1989). We calibrated the method by using seven stars of known $v \sin i$. The cross correlation method gives reliable results in the $5-60 \mathrm{~km} \mathrm{~s}^{-1}$ range. For values higher than $60 \mathrm{~km} \mathrm{~s}^{-1}$ the Gaussian fit we used is no longer adequate and the rotational broadening of the lines represent a large fraction of the observed spectral range. For values smaller than $5 \mathrm{~km} \mathrm{~s}^{-1}$ the intrinsic lines width is larger than the rotational broadening. The error for high signal-to-noise spectra is of the order of $\pm 2 \mathrm{~km} \mathrm{~s}^{-1}$. Further details on the method are given in Tagliaferri et al. (1999). The rotational data are given in Table 2.

\subsection{Inferred spectral classification}

As in the case of the EXOSAT and Einstein samples, for most of the observed stars the spectral classification is not well-defined. In order to infer or further constrain the spectral type and the luminosity class, we used our multicolour photometry, our estimate of RV and the intensity of the Ca I $6717.7 \AA$ line from our high resolution spectra, while the distances were taken from the HIPPARCOS catalogue (Perryman et al. 1997). Color indices of active stars have to be taken prudently when used for spectral classification, as the presence of activity phenomena can modify them by an unknown amount. Cutispoto et al. (1996) 
Table 4. List of the lines used for RV computations

\begin{tabular}{|c|c|c|c|c|c|}
\hline \multicolumn{2}{|c|}{ Li region } & \multicolumn{2}{|c|}{ H $\alpha$ region } & \multicolumn{2}{|c|}{ Ca II region } \\
\hline Elemen & $\lambda(\AA)$ & Element & $\lambda(\AA)$ & Element & $\lambda(\AA)$ \\
\hline Fe I & 6677.997 & FeI & 6533.970 & CrI & 3916.244 \\
\hline $\mathrm{Al} \mathrm{I}$ & 6696.032 & $\mathrm{FeI}+\mathrm{TiI}$ & 6546.252 & $\mathrm{FeI}$ & 3916.737 \\
\hline Fe Ip & 6696.322 & TiI & 6554.238 & $\mathrm{FeI}+\mathrm{CoI}$ & 3917.154 \\
\hline Al I & 6698.669 & SiI & 6555.466 & $\mathrm{FeI}+\mathrm{FeI}$ & 3918.612 \\
\hline Fe I & 6699.136 & TiI & 6556.077 & $\mathrm{FeI}+\mathrm{CrI}$ & 3919.119 \\
\hline Fe I & 6703.576 & $\mathrm{FeI}$ & 6556.806 & $\mathrm{FeI}$ & 3920.269 \\
\hline Fe I & 6705.105 & TiII & 6559.576 & FeI & 3922.923 \\
\hline Li I & 6707.815 & SiI & 6560.555 & TiI & 3924.533 \\
\hline Fe I & 6710.323 & $\mathrm{H} \alpha$ & 6562.808 & FeI & 3925.209 \\
\hline Fe I & 6713.044 & $\mathrm{CaI}$ & 6572.795 & FeI & 3925.651 \\
\hline Fe I & 6713.745 & $\mathrm{FeI}$ & 6574.254 & $\mathrm{FeI}+\mathrm{FeI}$ & 3925.988 \\
\hline Fe I & 6715.386 & FeI & 6575.037 & FeI & 3927.933 \\
\hline Fe I & 6716.252 & NiI & 6580.233 & CrI & 3928.644 \\
\hline Ca I & 6717.687 & FeI & 6581.218 & TiI & 3929.885 \\
\hline Sun & 6719.620 & SiI & 6583.710 & FeI & 3930.308 \\
\hline Si I & 6721.844 & NiI & 6586.310 & CaII K & 3933.682 \\
\hline Fe I & 6725.364 & CI & 6587.622 & FeI & 3937.336 \\
\hline Fe I & 6726.673 & FeI & 6591.326 & TiI & 3938.018 \\
\hline Fe I & 6729.050 & NiI & 6592.522 & MgI & 3938.409 \\
\hline Fe I & 6732.068 & $\mathrm{FeI}$ & 6592.926 & $\mathrm{FeI}$ & 3940.041 \\
\hline Fe I & 6733.153 & $\mathrm{FeI}$ & 6593.884 & $\mathrm{FeI}$ & 3941.284 \\
\hline Fe I & 6737.978 & FeI & 6597.571 & $\mathrm{CrI}$ & 3941.496 \\
\hline Fe I & 6739.524 & NiI & 6598.611 & FeI & 3942.448 \\
\hline Si I & 6741.629 & & & MnI & 3942.844 \\
\hline Ti I & 6743.127 & & & $\mathrm{FeI}$ & 3943.182 \\
\hline S I & 6743.575 & & & $\mathrm{FeI}$ & 3943.348 \\
\hline & & & & AlI & 3944.016 \\
\hline & & & & FeI & 3947.002 \\
\hline & & & & FeI & 3947.538 \\
\hline & & & & TiI & 3947.778 \\
\hline & & & & FeI & 3948.109 \\
\hline & & & & $\mathrm{TiI}+\mathrm{FeI}$ & 3948.733 \\
\hline & & & & FeI & 3949.141 \\
\hline & & & & FeI & 3949.959 \\
\hline & & & & $\mathrm{FeI}$ & 3951.171 \\
\hline
\end{tabular}

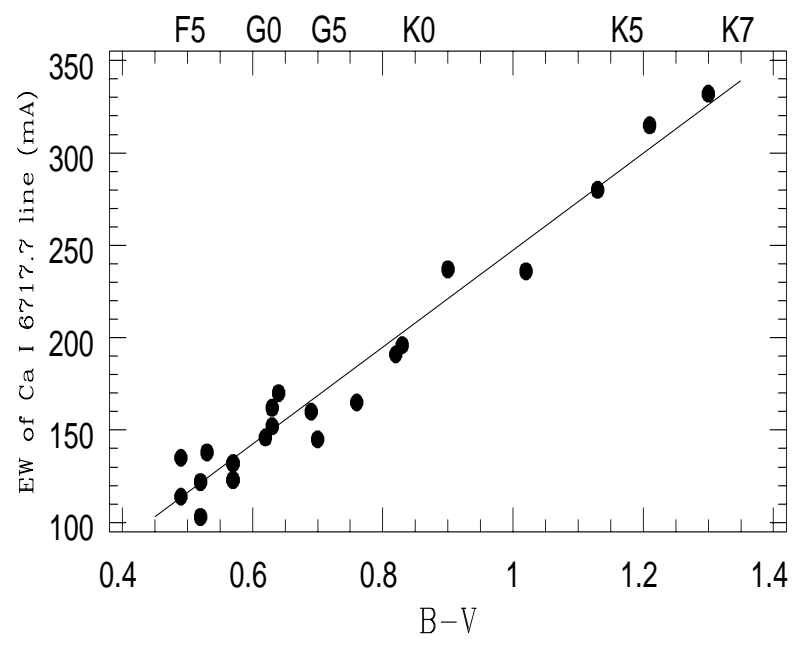

Fig. 1. Plot of the measured Ca I $6717.7 \AA$ line EW (m against the $B-V$ for a sample of 21 stars. The continuous line is the fit of the data developed a method, hereafter referred to as Active Star Colors (ASC), to infer the spectral classification from the observed colors. The ASC method was improved taking into account the effects of stellar activity on the $U-B$ index and considering the luminosity calibration of the HR diagram obtained by using the HIPPARCOS data (see Cutispoto 1998 and references therein for details). For stars later than K5 we used the luminosity calibration reported by Henry et al. (1998). The ASC method is better suitable for statistical purposes and for single stars. In the case of binary stars, two or more solutions can often reproduce the observed colors. However, if an accurate stars' distance measurement is available, it is possible to exclude most of the multiple solutions. Moreover, by using our spectra we can further constrain the allowed solutions. In particular, we used the Ca I $6717.7 \AA$ line as a spectral classification indicator. We first calibrated the equivalent width (EW) values of the Ca I $6717.7 \AA$ line vs. $B-V$. For this calibration we used the stars in our sample that are either single or have a WD companion, which give a negligible contribution to the continuum in the spectral region around the Ca I $6717.7 \AA$ line. Following these criteria, we selected 21 stars, 20 of which belonging to our sample plus HD 143937B $(B-V=1.30, \mathrm{EW}(\mathrm{Ca})=332$, $\mathrm{K} 7 \mathrm{~V})$. The latter star is the third component of the visual binary HD 143937, which is part of our sample. The measured Ca I $6717.7 \AA$ EW for the stars in our sample are reported in the last column of Table 2, where the value used for the EW vs. $B-V$ calibration are marked with "e". A double value means that we have measured both EWs of the SB2 components. We obtained a very good correlation between the EW and the color index $B-V$ (Fig. 1), being the scatter due either to errors in the measured EWs or to slight differences in metallicity among the stars. From the data in Fig. 1 we get the calibration: $B-V=0.00381 * \mathrm{EW}(\mathrm{Ca})+0.0577$ with a linear correlation coefficient $r=0.978$. We estimated a statistical error of $\pm 15 \mathrm{m \AA}$ for the EW values. The spectral classification is thus determined with an accuracy of \pm 2 spectral subclasses. The use of the Ca I $6717.7 \AA$ line EW was particularly important for the classification of the binary stars, allowing us to discern, by choosing the one that better fit the combined EW, between different possible solutions for both the ASC method and the distance. We are confident that the spectral types reported in Table 1 are very reliable, with uncertainties of the order of few spectral subclasses.

\section{Discussion of individual stars}

We discuss now the results for the individual stars. Our classification work has greatly benefited from the data collected by the HIPPARCOS satellite and presented by Perryman et al. (1997). In particular, for all stars the trigonometric parallax was often a key parameter for choosing between possible spectral classifications. 
Moreover, the magnitude difference between the components was very useful when studying visual binaries. Finally, the data from the variability annex to the HIPPARCOS catalogue (Perryman et al. 1997) were used for studying some of the variable stars.

HD 2133: Burleigh et al. (1997) classified this binary system as F9V + WD. However, our colors and HIPPARCOS distance are better matched by assuming a slightly evolved primary star. The constant RV indicates that the primary component is single.

HD 6628 = CS Cet: the colors are better matched by a binary system, in agreement with the HIPPARCOS distance. We have indication of binary nature also from the RVs deduced by two high resolution spectra separated by three days. Only one system of lines is visible. The star is given as a suspected RS CVn by McGale et al. (1995). Optical variability was clearly detected, but the period appears to be longer than the observing window, in agreement with the $v \sin i$ and the luminosity class IV classification of the primary component. A flare event was detected at HJD $=2449316.6145$.

HD 8357 = AR Psc: a well-known RS CVn-type variable, see Fekel (1996) and Cutispoto (1998) for the spectral classification. Our RV data are in agreement with the RV curve of Fekel (1996). We have no indication of SB2 nature.

HD 8358 = BI Cet: a well-known RS CVn-type variable with similar components, both very fast rotators (Bopp et al. 1985). Due to the very high rotation rate the spectrum is difficult to interpret. However, we clearly detect the two Ca I $6717.7 \AA$ lines.

HD $9770=$ BB Scl: a visual triple system. Components A and $\mathrm{B}$ are separated by 0.16 arcsec. Component $\mathrm{C}$ is 0.21 arcsec apart. Component B is also an eclipsing binary and is the most likely counterpart of the X-ray and EUV source (Cutispoto et al. 1997a). Due to its very fast rotation component $\mathrm{B}$ produces only clear depressions of the continuum in correspondence of the most prominent lines. The sharp lines that we see in our spectra are due to the single brighter A component. The spectral types of the $\mathrm{A}(\mathrm{K} 0 / 1 \mathrm{~V})$ and $\mathrm{B}$ $(\mathrm{K} 3 \mathrm{~V}+\mathrm{K} 3 / 4 \mathrm{~V})$ components, reported in Table 1 , are slightly revised with respect to the classification given in Cutispoto et al. (1997a).

HD 16699: at this position the SIMBAD database lists three stars separated by about 1.5 and 8.7 arcsec, with spectral type $\mathrm{F} 8 \mathrm{IV} / \mathrm{V}, \mathrm{K}$ and $\mathrm{K} 5$, respectively. The first two are components A and B of HD 16699, the third is SAO 232842. We obtained combined optical photometry for HD 16699AB + SAO 232842 and separate spectra for HD 16699 AB and SAO 232842. The spectral types that better reproduce the observed colors, the distance and the $\Delta V=0.57$ magnitude difference between HD 16699AB and SAO 232842 reported by HIPPARCOS are $\mathrm{F} 9 \mathrm{~V}+\mathrm{K} 0: \mathrm{V}$ and G1/2V for HD 16699AB and SAO 232842, respectively. In the spectrum of HD $16699 \mathrm{AB}$ we clearly see a system of sharp lines that we attribute to component $\mathrm{A}$, with a superimposed broader system of lines that we attributed to component B. Constant RV indicates that HD 16699AB is not a close binary. Also the RV of SAO 232842, for which we also measured a $v \sin i$ of $21 \pm 2 \mathrm{~km} \mathrm{~s}^{-1}$, is constant. The colors of HD 16699AB are also consistent with the classification $\mathrm{G} 2 / 3 \mathrm{~V}+\mathrm{G} 2 / 3 \mathrm{~V}$, that implies a distance of 62 pc. However, this is not consistent with the spectral signatures.

HD 18131: Burleigh et al. (1997) and Vennes et al. (1998) classified this binary system as K0IV + WD, in good agreement with our results. However, the K star does not have indication of strong activity (see also Osten \& Saar 1998). Therefore, the X-ray and EUV emission is most likely to be due to the WD. The RV measurements indicate that the system is not a close binary. The photometric distance results within the $92-120$ pc range measured by HIPPARCOS.

HD 24916: a visual binary whose components are separated by 11.1 arcsec. The inferred spectral types are those that better fit the colors, the distance and the magnitude difference $(\Delta V \sim 3.2)$ between the two components. The $\mathrm{K} 4 / 5 \mathrm{~V}$ star shows no indications of activity, therefore the X-ray and EUV emissions are assigned to the active M2Ve stars (see also Hodgkin \& Pye 1994). The RV and $v \sin i$ data refer to the K4/5V component.

HD 25457: a high proper motion star. The colors (Bessel 1990) and the HIPPARCOS distance better agrees with an F5/6V spectral classification. Our RV measurements seem to give a constant value and also the CORAVEL data (Duquennoy \& Mayor 1991) were not able to ascertain clear variability.

HD 32008: a binary system including a WD. Landsman et al. (1993) and Vennes et al. (1998) classified the primary as a K0IV and a G5IV star, respectively. However, both the colors reported in the literature and the HIPPARCOS distance are in better agreement with a G7IV/III classification. The primary star does not show indications of activity, therefore, the X-ray and EUV emissions are most likely to be due to the WD companion (see also Barstow et al. 1994). The RV data indicate that this system is not a close binary.

HD 33262: can be classified as an F7V star from the observed colors (Bessel 1990). The photometric distance is not in full agreement with the HIPPARCOS data. A metallicity value $[\mathrm{Fe} / \mathrm{H}]=-0.23$ was obtained by Cayrel de Strobel et al. (1992).

HD 35114: a very fast rotator for which we detected optical variability (Fig. 2). The period search analysis gives four possible values (i.e. $2.26 \pm 0.04,1.80 \pm 0.02$, 
$1.375 \pm 0.015$ and $0.690 \pm 0.004$ days). However, due to the very high $v \sin i$ only the shortest of them yields a steller radius that is compatible with the inferred spectral type. The photometric distance is somewhat larger than the value measured by HIPPARCOS.

HD 36869: we discovered optical variability (Fig. 3) with a period of $1.31 \pm 0.01$ days. The trigonometric parallax is from the Tycho catalogue. The distance, computed from the listed error, results in the $28-46 \mathrm{pc}$ range.

HD $37572=$ UY Pic: optical variability with a 4.52 day photometric period was reported in the SAAO Annual Report (1993) and is confirmed by us (Fig. 4). Soderblom et al. (1998) classified this star as ZAMS or younger. They obtained a $v \sin i$ and an RV that are both consistent with our values. HD 37572 is also a visual binary. The secondary component, SAO 217429, that lies about 18.3 arcsec apart, is not included in the photometry. From the magnitude difference measured by HIPPARCOS $(\Delta V \sim 1.9)$ we infer a K6:V spectral type for SAO 217429.

HD 41824: a close visual binary, with an angular separation of about 2.59 arcsec (Horch et al. 1997), whose primary component is a spectroscopic binary (Andersen et al. 1985), as confirmed by our RV measurements. We computed the spectral type of both the spectroscopic companion $(\mathrm{K} 7: \mathrm{V})$ and the visual companion $(\mathrm{G} 6 \mathrm{~V})$. The solution listed in Table 1 is the one that better fits simultaneously the distance and the magnitude difference $(\Delta V \sim 0.36)$ measured by HIPPARCOS, the observed colors and the spectral signatures. Our photometric observations (Fig. 5) also revealed low amplitude optical variability with a period of $3.3 \pm 0.1$ days.

HD 43162: low amplitude optical variability with a period of $7.2 \pm 0.2$ days was detected by us (Fig. 6). Our RV measurements are in good agreement with those of Beavers \& Eitter (1986) and Andersen et al. (1985) and indicate, within the errors, that the star is indeed single.

HD 43989 = V1538 Ori: a variable star discovered by Cutispoto et al. (1995) that computed a photometric period of 3.63 days. However, such a period would imply a minimum radius of the order of $3.4 R_{\odot}$, that is not consistent with the luminosity class $\mathrm{V}$ ensured by the HIPPARCOS distance. Hence, we performed a new period search and found that a period of $1.15 \pm 0.01$ days fits equally well (Fig. 7) the data collected by Cutispoto et al. (1995). This shorter period yields a minimum radius that is consistent with an F9V star seen at an inclination of about 75 degrees. Our RV data show that the star is single. Osten \& Saar (1998) inferred the two possible spectral classifications G0IV + G0IV and G0IV, however, from the above results, none of them can be accepted.

HD $45081=$ AO Men: was detected as un unsolved variable (i.e. a variable whose period has not been determined) by the HIPPARCOS satellite. Our observations (Fig. 8) are consistent with a $2.65 \pm 0.04$ day period. The photometric distance is smaller than the trigonometric one. This could be due to the fact that the star is probably a PMS object, as indicated by the inferred $0.9 R_{\odot}$ minimum radius. In fact, a $\mathrm{K} 5 \mathrm{~V}$ star should have a radius of the order of $0.68 R_{\odot}$. Actually, the period search analysis gives also a less significative peak corresponding to a photometric period of $0.722 \pm 0.003$ days. However, by using this shorter period the inclination would result in 21 degrees, a value that seems too small to account for the rather large amplitude of the light curve we observed.

HD 48189: a visual binary, whose components are separated by 0.76 arcsec, that are listed as a young, possibly PMS, star by Sterzik \& Schmitt (1997). Schachter et al. (1996) and Jeffries \& Jewell (1993) report the spectral types G0V + G8V and G0V + K3V, respectively. Our classification is in very good agreement with the latter and also fits the magnitude difference between the two components $(\Delta V \sim 2.3)$ measured by HIPPARCOS. We note that our data are not consistent with a PMS classification, as also noted by Micela et al. (1997). Both Andersen et al. (1985) and our RV data confirm that the primary G0V component is single. However, there is a difference between the mean values of the two RV data sets, that we think is due to the long-term effect due to the distant K2/3V secondary component. Finally, low amplitude light variability (Fig. 9) with a period of $2.60 \pm 0.04$ days was detected.

HD 71285: has an high rotation rate and is an SB system, as shown by our RV measurement. From the colors and the HIPPARCOS distance we infer a G1V + G3V classification. Optical variability (Fig. 10) with a period of $1.35 \pm 0.01$ days was discovered. Although the period search analysis gives also periods of 1.83 and 3.65 days, these longer values are non consistent with a dwarf classification and, hence, with the distance.

HD 75997: has a very fast rotation rate, as confirmed also by our photometric data (Fig. 11) that revealed optical variability with a period of $0.4085 \pm 0.0011$ days. The trigonometric parallax from HIPPARCOS has a large error, falling in the $22-55 \mathrm{pc}$ range.

HD 78644: is an SB1 system, as revealed by our RV measurements, and has a very high rotation rate. Optical variability (Fig. 12) with a photometric period of $0.840 \pm 0.004$ days was discovered. The inclination angle results of the order of 82 degrees and, in fact, the photometric data suggest the presence of an eclipse. Actually, the period search analysis gives also the photometric period of $0.551 \pm 0.002$ days, but such shorter period would led to an inclination of about 40 degrees, making quite difficult to explain the shape of the light curve. 
HD 82159: is a fast rotator and, as inferred by our RV measurements, is an SB1 system. The HIPPARCOS distance has a quite large error, falling into the $37-65$ pc range. We have not performed photometric observations of this star, however, from the spectral characteristics and the $B-V$ from the literature we infer a $\mathrm{G} 9 \mathrm{~V}+\mathrm{K} 4: \mathrm{V}$ classification. There is a second star, SAO $98614(V=8.67)$, at about 13.9 arcsec, that has a $v \sin i$ of $6 \mathrm{~km} \mathrm{~s}^{-1}$. For this star we infer a $\mathrm{K} 0: \mathrm{V}$ spectral type. The resulting distance of $36 \mathrm{pc}$ is in good agreement with the HIPPARCOS value, that falls into the $29-49 \mathrm{pc}$ range.

HD 82558 = LQ Hya: a well-known very active rapidly rotating single star that has been classified as a very young star just arrived on the ZAMS, or even as a PMS object (see Cutispoto 1998 and references therein).

HD 96064: a high proper-motion star that is also a triple visual system. Components $\mathrm{B}$ and $\mathrm{C}$, which are 0.4 arcsec apart, lay 11.5 arcsec aside component A. We observed component A, which is an active G8V star showing low amplitude optical variability with a period of $6.9 \pm 0.3$ days (Fig. 13). Components $\mathrm{B}(\mathrm{K} 9: \mathrm{V})$ and $\mathrm{C}(\mathrm{K} 7: \mathrm{V})$ constitute a very close pair of late type stars, whose spectral types were inferred from the magnitude difference with respect to component A reported by HIPPARCOS.

HD 118100 = EQ Vir: a well-known flare star.

HD 124672: we find it to be a short period variable star with a photometric period of $0.646 \pm 0.003$ days (Fig. 14). The best fit of the colors is obtained by assuming an F5V + K4:V system, however, the spectral lines are too broad to give any further indication on the secondary component, whose spectral type is very uncertain.

HD 140637 = KW Lup: a WTTS star and also a visual binary with a separation of 0.67 arcsec and a very faint companion (Brandner et al. 1996). It was classified as a 11 Myr star with a mass of $1 M_{\odot}$ by Neuhäuser \& Brandner (1998). Our photometry confirms the PMS nature of this object. In fact, the colors are not consistent with any class $V$ star with a $B-V$ close to the value expected for a $\mathrm{K} 3 \mathrm{~V}$ and a $V-I$ similar to the value expected for a K5V. Moreover, if we assume a main sequence star the distance would be smaller than the value reported by HIPPARCOS. HD 140637 is listed as a semi-regular variable with an amplitude of about 0.16 magnitudes in the HIPPARCOS variability annex. Our observations (Fig. 15) are consistent with a $2.72 \pm 0.05$ day period. The RV is constant in three spectra taken in consecutive nights. This indicates that HD 140637 is effectively single, the effects due to the optical companion being not detectable over a three night interval.

HD 141943: two photometric periods are possible. The shorter one $(1.30 \pm 0.01$ days $)$ implies a luminosity class $\mathrm{V}$ classification and a distance of $46 \mathrm{pc}$. The longer one $(2.20 \pm 0.03$ days, see Fig. 16) yields a larger radius, adequate for a PMS or an evolved object. Although further photometric observations are needed to ascertain the true rotational period we regard this star as a PMS, due to its high rotational rate and to the high Li abundance inferred by Tagliaferri et al. (1999).

HD 143937 = V1044 Sco: a visual binary with an angular separation of 10.25 arcsec. We find that the brighter A component is an SB2 and Cutispoto (1999) discovered that it is also an eclipsing binary with an orbital period of about 0.91 days. Our photometric observations (Fig. 17) included both components of the visual binary. The fainter B component has a $v \sin i$ of $12 \pm 3 \mathrm{~km} \mathrm{~s}^{-1}$. The best fit of the observed colors, spectral characteristics and distance is obtained by assuming the eclipsing binary as a G9V + $\mathrm{M} 0: \mathrm{V}$ system and component $\mathrm{B}$ as a $\mathrm{K} 7: \mathrm{V}$ star. This is also in agreement with the $\Delta V \simeq 2.8 \mathrm{mag}$ nitude difference between $\mathrm{A}$ and $\mathrm{B}$ components measured by HIPPARCOS. The data reported in Tables 1 and 2 refer to the out-of-eclipse light curve. The star is also reported as an unsolved variable in the HIPPARCOS variability annex.

HD $156498=$ V2369 Oph: a close visual pair $(\rho=0.31$ arcsec, $\Delta V \simeq 1.4$ ) whose primary component is an SB1 binary (Jeffries et al. 1995), as also confirmed by our spectra. The spectral classification that is reported in Table 1 is the only one that reproduce the observed colors, the HIPPARCOS distance and the magnitude difference between the components. HD 156498 is listed in the HIPPARCOS variability annex as an unsolved variable with an amplitude of 0.08 magnitudes. Our observations (Fig. 18) show that the optical variability is consistent with the 0.655 -day orbital period given by Jeffries et al. (1995). The G8:V B component has a $v \sin i$ of only $8 \pm 2 \mathrm{~km} \mathrm{~s}^{-1}$.

HD 171488 = V889 Her: was discovered to be a short period variable by Henry et al. (1995), who reported a photometric period of 1.338 days and an amplitude of about 0.1 magnitudes in the $V$-band. For this star we only get few and sparse photometric data. However, the colors and the spectral signatures are consistent with a $\mathrm{G} 2 \mathrm{~V}$ classification, that results in very good agreement with both the distance measured by HIPPARCOS and the classification inferred by Osten \& Saar (1998). The RV is constant, in agreement with the results of Fekel (1997). From the strength of the Li line Mullis \& Bopp (1994) estimated for HD 171488 an age similar to or younger than the Pleiades.

HD $195434=$ MR Del: a visual binary $(\rho=1.80$ arcsec, $\Delta V \simeq 0.28)$ whose primary component $(\mathrm{K} 2 \mathrm{~V}+\mathrm{K} 6 \mathrm{~V})$ is an eclipsing binary with an orbital period of 0.52175 days, as first observed by Cutispoto et al. (1997a). It 
is a high proper-motion very old star which is still very active because of its tidally locked high rotation rate. For a detailed study see Cutispoto et al. (1997a). Here we note that there is a typo in the spectral classification given in Table 1 of the above-mentioned paper. The correct classification is the one reported in Table 2 of the present paper. The star is also present in the HIPPARCOS variability annex, where a period of 0.521690 days is listed.

HD 197890 = BO Mic: with a photometric period of 0.380 days (see Cutispoto et al. $1997 \mathrm{~b}$ and references therein) it is the most rapidly rotating nearby single late-type star known to date. The spectral classification from the colors is quite difficult, probably owing to the very high level of activity and/or to the fact that probably HD 197890 has not arrived on the MS, yet. From the $v \sin i=170 \mathrm{~km} \mathrm{~s}^{-1}$ computed by Anders et al. (1993) and the photometric period, Cutispoto et al. (1997b) inferred a minimun radius in the $1.13-1.43 R_{\odot}$ range, thus supporting the hypothesis that HD 197890 is a PMS star, as also confirmed by the now available HIPPARCOS distance. The photometric period reported in the HIPPARCOS variability annex is in full agreement with the value given by Cutispoto et al. (1997b). The equivalent width of the Ca I $6717.7 \AA$ line suggests a spectral type $\mathrm{K} 5$, later than the $\mathrm{K} 2 / 3$ proposed by the photometry. However, the noise of the spectra and the very high $v \sin i$ of the star make the measurement of the Ca I line very difficult and hence the EW of the line is probably overestimated.

HD 199143: the classification from the observed colors and the spectral signatures result in good agreement with the distance measured by HIPPARCOS. Very low amplitude optical variability is possible.

HD 217411: has a WD companion as reported by Barstow et al. (1994) and by Vennes et al. (1998). The primary component shows only moderate signs of activity. It is a slow rotator (see also Fekel 1997), has no Ca II emission and shows only a partially filled $\mathrm{H} \alpha$ line (Mullis \& Bopp 1994). We do not see indications of RV variability, in agreement with Fekel (1997), hence the system is a wide binary. However, it should be noted that for this star we observe an anomalous strength of the Ca I $6717.7 \AA$ line, that would be consistent with being the primary component of HD 217411 a G2/3V $+\mathrm{K} 4 \mathrm{~V}$ system. On the other hand this circumstance appears in contrast with the RV measurements.

HD $222259=$ DS Tuc: is a close visual binary $(\rho=5.3$ arcsec, $\Delta V \sim 1.3)$ that is reported as an unsolved variable with an amplitude of 0.12 magnitudes in the HIPPARCOS variability annex. Our photometry, in which the two components were observed together, shows a much smaller amplitude light curve (Fig. 19) with a period of $1.54 \pm 0.02$ days. This system was also studied by Soderblom et al. (1998).
HD 223816: was classified as a G0V + WD system by Barstow et al. (1994). Our colors agree best with those of an F5V star. This earlier classification for the primary component implies for the WD a temperature lower than the value computed by Barstow et al. (1994). Similarly to our results, also Vennes et al. (1998) observed a constant RV on short timescale. The difference among the two data sets could be due to the long-term variability caused by the presence of the WD. Finally, Craig et al. (1997) classified the WD as metal-rich and the primary component as F5IV. However, we note that the latter classification gives a worse fit of the observed color than the F5V one. The best fit of the colors by assuming a class IV star, still somewhat worse than the fit with the F5V, is obtained by a F6/7 IV, whose distance results in $195 \mathrm{pc}$.

HD 291095 = V1355 ori: was found to be a variable star by Cutispoto et al. (1995), who computed a photometric period of 3.82 days and inferred, from the observed colors, the two possible spectral classifications $\mathrm{K} 2 / 3 \mathrm{~V}$ and $\mathrm{K} 2 \mathrm{IV}+\mathrm{G} 2 \mathrm{~V}$. More recently, this star was studied by Osten \& Saar (1998) who rule out the dwarf classification. Given the $23-133$ pc distance range measured by HIPPARCOS, we revise the spectral types listed by Cutispoto et al. (1995) to K1 V/IV $(d=88 \mathrm{pc})$ and $\mathrm{K} 2 \mathrm{~V} / \mathrm{IV}+\mathrm{G} 8 \mathrm{~V}(d=100 \mathrm{pc})$, respectively. However, we note that also the combination $\mathrm{K} 3 \mathrm{~V} / \mathrm{IV}+\mathrm{G} 6 \mathrm{~V}(d=102 \mathrm{pc})$ fits the observed colors. The RV measurements indicate that the star is indeed a binary, thus, we prefer the latter spectral classification because it better agrees with the minimum radius inferred for the primary component.

BD-00 1712: is an SB2 with an orbital period of 1.4 days (Jeffries et al. 1995). Optical variability with a photometric period of 1.412 days is reported by Robb \& Gladders (1996). For this star we only got few and sparse photometric data. However, the colors and the spectral signatures are in agreement with a $\mathrm{K} 3 \mathrm{~V}+$ M0:V classification. Ca II $\mathrm{K}$ and $\mathrm{H} \alpha$ emission lines have been detected by Jeffries et al. (1995).

BD $+08102=$ BL Psc: has been extensively studied by Kellett et al. (1995) who report a K2V spectral type, optical variability with several possible photometric periods, $v \sin i=90 \mathrm{~km} \mathrm{~s}^{-1}$, the absence of $\mathrm{Li}$ and the presence of a WD companion at about 2 arcsec. Our photometric and spectroscopic data are in full agreement with these results, with the photometric observations (Fig. 20) in agreement with the 0.2937 day period found by Kellett et al. (1995). However, Kellett et al. (1995) also speculate that more than $90 \%$ of the EUV flux is due to the WD and that the two stars form a wide binary system with the high rotation rate of the $\mathrm{K}$ star explained by its young age. These conclusions are in contrast with: a) the $\mathrm{K}$ star is an extreme active fast rotator and as such it must 
Table 5. Star name, HJD $(2449000.0+)$, heliocentric RV and error, number of lines used $(N)$, spectral region $(\mathrm{SR})$, notes

\begin{tabular}{|c|c|c|c|c|}
\hline Star name & HJD & $\mathrm{RV}$ & $\bar{N}$ & SR notes \\
\hline \multirow{2}{*}{ HD 2133} & 956.8198 & $-2.9 \pm 0.6$ & 6 & $\mathrm{LiC}$ \\
\hline & 957.7336 & $-2.3 \pm 1.5$ & 5 & $\mathrm{Ha}$ \\
\hline \multirow[t]{2}{*}{ HD 6628} & 740.5366 & $42.1 \pm 1.4$ & 16 & Li $\quad$ SB1 \\
\hline & 743.5551 & $46.1 \pm 0.8$ & 14 & $\mathrm{Ha}$ \\
\hline \multirow[t]{3}{*}{ HD 8357} & 954.8141 & $19.4 \pm 0.8$ & 18 & $\overline{\mathrm{Li}} \mathrm{SB1}$ \\
\hline & 957.8206 & $46.2 \pm 1.0$ & 20 & $\mathrm{Ha}$ \\
\hline & 960.7194 & $18.0 \pm 2.5$ & 13 & $\mathrm{Ca}$ \\
\hline \multirow[t]{2}{*}{ HD 8358} & 955.7220 & $-11.3 \pm 4.8$ & 3 & Li SB2 \\
\hline & 958.8292 & $80.0 \pm 1.0$ & 1 & $\mathrm{Ha}$ \\
\hline \multirow[t]{6}{*}{ HD $9770 \mathrm{~A}$} & 738.5344 & $31.3 \pm 0.5$ & 19 & $\mathrm{Li} \mathrm{C}$ \\
\hline & 738.5498 & $31.5 \pm 0.2$ & 14 & $\mathrm{Li}$ \\
\hline & 738.5913 & $31.5 \pm 0.3$ & 12 & $\mathrm{Li}$ \\
\hline & 739.5564 & $31.6 \pm 0.3$ & 11 & $\mathrm{Li}$ \\
\hline & 741.5558 & $31.2 \pm 0.8$ & 12 & $\mathrm{Ha}$ \\
\hline & 744.5317 & $31.8 \pm 1.0$ & 7 & $\mathrm{Ca}$ \\
\hline \multirow[t]{3}{*}{ HD $16699 \mathrm{~A}$} & 955.7593 & $16.4 \pm 0.7$ & 17 & $\mathrm{Li} C$ \\
\hline & 957.7746 & $16.2 \pm 0.9$ & 19 & $\mathrm{Ha}$ \\
\hline & 959.8114 & $15.9 \pm 1.1$ & 28 & $\mathrm{Ca}$ \\
\hline \multirow[t]{3}{*}{$\overline{\mathrm{SAO}} 232842$} & 955.7849 & $16.1 \pm 0.7$ & 11 & $\mathrm{Li} \quad \mathrm{C}$ \\
\hline & 957.7593 & $16.4 \pm 2.6$ & 11 & $\mathrm{Ha}$ \\
\hline & 959.7881 & $15.6 \pm 2.1$ & 7 & $\mathrm{Ca}$ \\
\hline \multirow[t]{3}{*}{ HD 18131} & 739.5375 & $11.4 \pm 0.8$ & 18 & $\mathrm{LiC}$ \\
\hline & 743.5858 & $11.8 \pm 0.7$ & 16 & $\mathrm{Ha}$ \\
\hline & 744.5607 & $10.9 \pm 1.4$ & 12 & $\mathrm{Ca}$ \\
\hline \multirow{2}{*}{ HD 24916} & 740.5886 & $3.3 \pm 0.6$ & 12 & $\mathrm{LiC}$ \\
\hline & 742.5732 & $3.7 \pm 0.5$ & 13 & $\mathrm{Ha}$ \\
\hline \multirow[t]{4}{*}{ HD 25457} & 738.6101 & $17.9 \pm 2.1$ & 12 & $\overline{\mathrm{Li}} \mathrm{C}$ \\
\hline & 740.5589 & $17.8 \pm 1.2$ & 8 & $\mathrm{Li}$ \\
\hline & 742.5525 & $18.4 \pm 1.2$ & 7 & $\mathrm{Ha}$ \\
\hline & 745.5269 & $17.4 \pm 2.0$ & 7 & $\mathrm{Ca}$ \\
\hline \multirow[t]{2}{*}{ HD 32008} & 740.6181 & $-15.8 \pm 0.9$ & 24 & $\mathrm{Li} \mathrm{C}$ \\
\hline & 741.5892 & $-15.3 \pm 0.4$ & 16 & $\mathrm{Ha}$ \\
\hline \multirow[t]{2}{*}{ HD 33262} & 738.6163 & $-1.3 \pm 0.7$ & 11 & $\mathrm{Li} C$ \\
\hline & 741.5933 & $-0.9 \pm 0.7$ & 5 & $\mathrm{Ha}$ \\
\hline \multirow[t]{3}{*}{ HD 36869} & 954.9112 & $24.1 \pm 0.6$ & 8 & $\mathrm{LiC}$ \\
\hline & 957.8541 & $23.5 \pm 0.7$ & 6 & $\mathrm{Ha}$ \\
\hline & 959.9019 & $24.8 \pm 2.0$ & 6 & $\mathrm{Ca}$ \\
\hline \multirow[t]{3}{*}{ HD 37572} & 740.6554 & $32.6 \pm 0.7$ & 17 & $\overline{\mathrm{Li}} \mathrm{C}$ \\
\hline & 742.6236 & $32.8 \pm 0.8$ & 20 & $\mathrm{Ha}$ \\
\hline & 744.6026 & $31.9 \pm 1.7$ & 22 & $\mathrm{Ca}$ \\
\hline \multirow[t]{3}{*}{ HD 41824} & 740.6991 & $13.3 \pm 1.0$ & 10 & $\begin{array}{ll}\mathrm{Li} & \mathrm{SB} 1\end{array}$ \\
\hline & 742.7423 & $4.2 \pm 1.1$ & 10 & $\mathrm{Ha}$ \\
\hline & 744.6358 & $16.7 \pm 1.8$ & 28 & $\mathrm{Ca}$ \\
\hline \multirow[t]{3}{*}{ HD 43162} & 740.7189 & $21.8 \pm 0.6$ & 21 & $\mathrm{LiC}$ \\
\hline & 742.7538 & $21.8 \pm 1.2$ & 20 & $\mathrm{Ha}$ \\
\hline & 745.5900 & $21.3 \pm 0.9$ & 34 & $\mathrm{Ca}$ \\
\hline \multirow[t]{3}{*}{ HD 43989} & 739.6029 & $21.9 \pm 1.9$ & 3 & $\mathrm{LiC}$ \\
\hline & 742.6807 & $25.7 \pm 2.7$ & 4 & $\mathrm{Ha}$ \\
\hline & 745.6142 & $20.5 \pm 5.8$ & 5 & $\mathrm{Ca}$ \\
\hline
\end{tabular}

have a very strong X-ray and EUV emission and b) the $\mathrm{K}$ star has a very low, if any, Li abundance and as such cannot be very young. The high rotation rate of the $\mathrm{K}$ star, instead, could be explained by tidally effects induced by close binarity. Indeed, there is a clear indication of RV variability in the two spectra we have of the $\mathrm{K} 2 \mathrm{~V}$ component.

BD+09 73 = BK Psc: a high proper-motion star. Due to a blue excess in the $U-B$ color, confirmed by us, the presence of a WD companion has been suggested by Weis (1991). Optical variability with a photometric
Table 5. continued

\begin{tabular}{|c|c|c|c|c|c|}
\hline Star name & HJD & RV & $\bar{N}$ & SR & notes \\
\hline \multirow[t]{2}{*}{ HD 45081} & 740.7559 & $16.3 \pm 1.0$ & 8 & $\mathrm{Li}$ & $\mathrm{C}$ \\
\hline & 743.7700 & $16.1 \pm 0.8$ & 9 & Ha & \\
\hline \multirow[t]{3}{*}{ HD 48189} & 738.7497 & $28.5 \pm 2.0$ & 6 & $\mathrm{Li}$ & $\mathrm{C}$ \\
\hline & 741.7140 & $29.4 \pm 1.0$ & 8 & $\mathrm{Ha}$ & \\
\hline & 745.6368 & $27.4 \pm 1.8$ & 5 & $\mathrm{Ca}$ & \\
\hline \multirow[t]{3}{*}{ HD 71285} & 739.8005 & $-20.8 \pm 1.9$ & 6 & $\mathrm{Li}$ & SB1 \\
\hline & 742.7818 & $71.3 \pm 1.7$ & 7 & Ha & \\
\hline & 744.7381 & $67.8 \pm 1.2$ & 5 & $\mathrm{Ca}$ & \\
\hline \multirow[t]{3}{*}{ HD 78644} & 739.8556 & $9.7 \pm 2.6$ & 3 & $\mathrm{Li}$ & SB1? \\
\hline & 743.8292 & $48.4 \pm 1.7$ & 5 & $\mathrm{Ha}$ & \\
\hline & 745.7968 & $-19.3 \pm 1.7$ & 4 & $\mathrm{Ca}$ & \\
\hline \multirow{3}{*}{ HD 82159} & 739.7137 & $51.7 \pm 0.9$ & 15 & $\overline{\mathrm{Li}}$ & SB1 \\
\hline & 742.7242 & $27.5 \pm 0.7$ & 15 & Ha & \\
\hline & 745.7083 & $3.8 \pm 1.1$ & 4 & $\mathrm{Ca}$ & \\
\hline \multirow[t]{3}{*}{ SAO 98614} & 739.7561 & $27.1 \pm 0.6$ & 21 & $\mathrm{Li}$ & $\mathrm{C}$ \\
\hline & 741.7519 & $27.0 \pm 1.0$ & 20 & $\mathrm{Ha}$ & \\
\hline & 745.7381 & $26.3 \pm 1.0$ & 1 & $\mathrm{Ca}$ & \\
\hline \multirow[t]{2}{*}{ HD 82558} & 740.8207 & $7.3 \pm 0.9$ & 7 & $\mathrm{Li}$ & $\mathrm{C}$ \\
\hline & 742.8273 & $7.1 \pm 1.7$ & 7 & $\mathrm{Ha}$ & \\
\hline \multirow[t]{4}{*}{ HD 96064} & 740.8659 & $18.8 \pm 0.8$ & 20 & $\mathrm{Li}$ & $\mathrm{C}$ \\
\hline & 742.8721 & $18.7 \pm 0.6$ & 16 & $\mathrm{Ha}$ & \\
\hline & 743.8826 & $18.3 \pm 0.6$ & 16 & Ha & \\
\hline & 745.8633 & $17.5 \pm 0.8$ & 16 & $\mathrm{Ca}$ & \\
\hline \multirow[t]{2}{*}{ HD 118100} & 738.8627 & $-22.0 \pm 0.8$ & 12 & $\mathrm{Li}$ & $\mathrm{C}$ \\
\hline & 741.8668 & $-22.4 \pm 0.8$ & 13 & $\mathrm{Ha}$ & \\
\hline \multirow[t]{3}{*}{ HD 140637} & 956.4957 & $-4.3 \pm 1.1$ & 18 & $\mathrm{Li}$ & $\mathrm{C}$ \\
\hline & 958.5357 & $-5.0 \pm 1.0$ & 12 & $\mathrm{Ha}$ & \\
\hline & 959.5109 & $-5.8 \pm 1.7$ & 4 & $\mathrm{Ca}$ & \\
\hline \multirow[t]{3}{*}{ HD 141943} & 955.5441 & $-0.5 \pm 2.5$ & 6 & $\mathrm{Li}$ & $\mathrm{C}$ \\
\hline & 957.5332 & $-4.0 \pm 2.3$ & 3 & Ha & \\
\hline & 959.5037 & $-1.7 \pm 3.1$ & 6 & $\mathrm{Ca}$ & \\
\hline \multirow[t]{6}{*}{ HD 143937A } & 954.5567 & $-155.2 \pm 2.0$ & 2 & $1 \mathrm{Li}$ & SB2 \\
\hline & 954.5567 & $52.5 \pm 6.6$ & 2 & $2 \mathrm{Li}$ & \\
\hline & 956.5923 & $-135.4 \pm 1.5$ & 2 & $1 \mathrm{Li}$ & \\
\hline & 956.5923 & $34.4 \pm 2.2$ & 2 & $2 \mathrm{Li}$ & \\
\hline & 960.5382 & $60.6 \pm 7.4$ & 2 & $1 \mathrm{Ca}$ & \\
\hline & 960.5382 & $-172.3 \pm 15.7$ & 2 & $2 \mathrm{Ca}$ & \\
\hline HD 143937B & 955.6130 & $-55.0 \pm 1.1$ & 8 & $\mathrm{Li}$ & \\
\hline \multirow[t]{3}{*}{ HD 156498} & 955.4837 & $20.6 \pm 0.5$ & 19 & $\mathrm{Li}$ & $?$ \\
\hline & 958.4783 & $14.4 \pm 0.6$ & 8 & $\mathrm{Ha}$ & \\
\hline & 958.4783 & $26.0 \pm 0.5$ & 8 & $\mathrm{Ha}$ & \\
\hline \multirow[t]{3}{*}{ HD 171488} & 954.4807 & $-22.1 \pm 1.1$ & 4 & $\mathrm{Li}$ & $\mathrm{C}$ \\
\hline & 957.4914 & $-22.7 \pm 2.9$ & 3 & $\mathrm{Ha}$ & \\
\hline & 959.4700 & $-25.8 \pm 4.8$ & 8 & $\mathrm{Ca}$ & \\
\hline \multirow[t]{3}{*}{ HD 195434} & 956.5663 & $-51.6 \pm 0.5$ & 16 & $\mathrm{Li}$ & $\mathrm{C}$ \\
\hline & 957.5176 & $-51.9 \pm 0.5$ & 14 & $\mathrm{Ha}$ & \\
\hline & 960.5993 & $-51.5 \pm 1.0$ & 20 & $\mathrm{Ca}$ & \\
\hline
\end{tabular}

period of 2.17 days was reported in the SAAO Annual Report (1993). Our observations (Fig. 21) confirm the optical variability but with a slightly longer period of $2.24 \pm 0.04$ days. The SB1 nature, already noted by Jeffries et al. (1995), is confirmed by our RV measurements. The best fit of the observed colors and HIPPARCOS distance is obtained by assuming a $\mathrm{K} 5 / 6: \mathrm{V}+\mathrm{M} 4: \mathrm{V}+\mathrm{WD}$ system.

SAO 91772 = LN Peg: was reported as a SB1 Pop II binary with an orbital period of 1.84422 days by Latham et al. (1988). Optical variability with a period of 1.84 days was discovered by Rodonò et al. (1994). The observations presented in this paper (Fig. 22) are consistent with a shorter $1.820 \pm 0.015$ day period. Pasquini 
Table 5. continued

\begin{tabular}{|c|c|c|c|c|c|}
\hline Star name & HJD & $\overline{\mathrm{RV}}$ & $N$ & SR & notes \\
\hline \multirow[t]{4}{*}{ HD 197890} & 954.5989 & $-11.3 \pm 10.8$ & 3 & $\mathrm{Li}$ & $\mathrm{C}$ \\
\hline & 954.6457 & $-16.7 \pm 11.5$ & 3 & $\mathrm{Li}$ & \\
\hline & 956.6620 & $-2.1 \pm 26.0$ & 3 & $\mathrm{Li}$ & \\
\hline & 959.6440 & $-17.6 \pm 6.2$ & 2 & $\mathrm{Ca}$ & \\
\hline \multirow{3}{*}{ HD 217411} & 954.6910 & $21.7 \pm 0.6$ & 26 & $\mathrm{Li}$ & $\mathrm{C}$ \\
\hline & 958.6669 & $21.4 \pm 0.6$ & 17 & $\mathrm{Ha}$ & \\
\hline & 959.7099 & $22.3 \pm 0.9$ & 20 & $\mathrm{Ca}$ & \\
\hline \multirow[t]{3}{*}{ HD222259 } & 955.6679 & $8.3 \pm 1.5$ & 10 & $\overline{\mathrm{Li}}$ & $\mathrm{C}$ \\
\hline & 957.6955 & $7.4 \pm 0.6$ & 8 & $\mathrm{Ha}$ & \\
\hline & 959.7603 & $7.3 \pm 2.5$ & 8 & $\mathrm{Ca}$ & \\
\hline \multirow[t]{3}{*}{ HD 223816} & 956.7708 & $20.3 \pm 2.2$ & 6 & $\mathrm{Li}$ & $\mathrm{C}$ \\
\hline & 958.8069 & $18.8 \pm 3.4$ & 5 & $\mathrm{Ha}$ & \\
\hline & 960.7263 & $18.0 \pm 3.0$ & 6 & $\mathrm{Ca}$ & \\
\hline \multirow{2}{*}{ HD 291095} & 738.6510 & $78.9 \pm 10.6$ & 9 & $\mathrm{Li}$ & SB1 \\
\hline & 741.6356 & $27.3 \pm 5.8$ & 4 & $\mathrm{Ha}$ & \\
\hline \multirow[t]{5}{*}{$\mathrm{BD}-001712$} & 739.6614 & $-80.2 \pm 3.2$ & 5 & $1 \mathrm{Li}$ & SB2 \\
\hline & 739.6614 & $100.9 \pm 1.0$ & 4 & $2 \mathrm{Li}$ & \\
\hline & 743.6641 & $5.5 \pm 1.1$ & 7 & $\mathrm{Ha}$ & \\
\hline & 745.6703 & $-29.7 \pm 1.0$ & 1 & $1 \mathrm{Ca}$ & \\
\hline & 745.6703 & $45.5 \pm 1.0$ & 1 & $2 \mathrm{Ca}$ & \\
\hline \multirow{2}{*}{$\overline{B D}+0973$} & 956.7180 & $-26.3 \pm 4.0$ & 11 & $\mathrm{Li}$ & SB1 \\
\hline & 958.7741 & $-40.6 \pm 0.8$ & 11 & $\mathrm{Ha}$ & \\
\hline \multirow[t]{3}{*}{ SAO 91772} & 954.7394 & $40.2 \pm 1.5$ & 7 & $\mathrm{Li}$ & SB1 \\
\hline & 958.7425 & $23.1 \pm 4.0$ & 12 & $\mathrm{Ha}$ & \\
\hline & 960.6814 & $8.7 \pm 3.5$ & 14 & $\mathrm{Ca}$ & \\
\hline \multirow[t]{3}{*}{ SAO 111210} & 955.8203 & $10.0 \pm 2.1$ & 7 & $\mathrm{Li}$ & $\mathrm{C}$ \\
\hline & 957.7972 & $11.0 \pm 2.3$ & 6 & $\mathrm{Ha}$ & \\
\hline & 959.8499 & $8.1 \pm 2.8$ & 6 & $\mathrm{Ca}$ & \\
\hline \multirow[t]{3}{*}{ SAO 150508} & 954.8750 & $26.6 \pm 0.8$ & 7 & $\overline{\mathrm{Li}}$ & $\mathrm{C}$ \\
\hline & 958.8513 & $25.6 \pm 1.3$ & 5 & $\mathrm{Ha}$ & \\
\hline & 960.8478 & $27.2 \pm 2.1$ & 6 & $\mathrm{Ca}$ & \\
\hline \multirow[t]{2}{*}{ SAO 150676} & 958.8798 & $25.8 \pm 1.4$ & 10 & $\mathrm{Li}$ & $\mathrm{C}$ \\
\hline & 956.8597 & $25.9 \pm 1.8$ & 5 & $\mathrm{Ha}$ & \\
\hline \multirow[t]{6}{*}{ SAO 151224} & 738.7098 & $79.3 \pm 5.3$ & 6 & $1 \mathrm{Li}$ & SB2 \\
\hline & 738.7098 & $-27.3 \pm 1.3$ & 5 & $2 \mathrm{Li}$ & \\
\hline & 743.7180 & $80.8 \pm 9.7$ & 5 & $1 \mathrm{Ha}$ & \\
\hline & 743.7180 & $-29.4 \pm 2.6$ & 9 & $2 \mathrm{Ha}$ & \\
\hline & 744.6897 & $96.3 \pm 1.0$ & 1 & $1 \mathrm{Ca}$ & \\
\hline & 744.6897 & $-34.9 \pm 1.0$ & 1 & $2 \mathrm{Ca}$ & \\
\hline \multirow[t]{3}{*}{ SAO 196024} & 960.9017 & $22.5 \pm 1.2$ & 17 & $\mathrm{Li}$ & $\mathrm{C}$ \\
\hline & 957.8961 & $21.7 \pm 1.2$ & 11 & $\mathrm{Ha}$ & \\
\hline & 956.9021 & $22.2 \pm 2.9$ & 17 & $\mathrm{Ca}$ & \\
\hline \multirow[t]{2}{*}{ SAO 234124} & $=955.8918$ & $\overline{75.2 \pm 2.5}$ & 18 & $\overline{\mathrm{Li}}$ & SB1 \\
\hline & 958.9080 & $-45.0 \pm 2.9$ & 11 & $\mathrm{Ha}$ & \\
\hline \multirow[t]{2}{*}{$\mathrm{SAO} 243278$} & 954.5192 & $-101.9 \pm 2.5$ & 3 & $1 \mathrm{Li}$ & SB2 \\
\hline & 954.5192 & $96.5 \pm 7.4$ & 3 & $2 \mathrm{Li}$ & \\
\hline
\end{tabular}

\& Lindgren (1994) estimated a magnitude difference $\Delta V \simeq 1.2$ between the two components from the Wilson-Bappu relation. The color observed by us also suggest the presence of a late-type companion. The best spectral classification that agrees with both the observed colors and the HIPPARCOS distance implies class $\mathrm{V}$ components, ruling out the subgiant classification reported, among others, by Ottmann et al. (1997). Finally, we note that the magnitude difference between the two components inferred by us is much larger than the value computed by Pasquini \& Lindgren (1994). More recentely Fekel et al. (1999) discovered that SAO 91772 is indeed a triple system.
SAO 111210: a visual binary system $(\rho=0.9$ arcsec, $\Delta V \simeq 1.5)$ whose components were observed together both in photometry and spectroscopy. A low rotation rate is inferred for the secondary star, whose spectral type is computed to fit both the observed colors and the magnitude difference between the two components. We discovered optical variability with a period of $0.5473 \pm 0.0010$ days (Fig. 23). Another possible photometric period is $0.844 \pm 0.002$ days.

SAO 150508: a star for which the HIPPARCOS satellite gives a distance in the $14-88 \mathrm{pc}$ range. Our inferred spectral classification is consistent with the upper limit. We discovered optical variability with a period of $1.90 \pm 0.01$ days (Fig. 24).

SAO 150676: a star for which the two RV measurements we have show no sign of binarity. The colors are consistent with a G2 V classification and optical variability, with a period of $1.78 \pm 0.01$ days (Fig. 25), has been discovered. Moreover, the very high $v \sin i$ implies a minimum radius of $1.0 R_{\odot}$, so that we suggest that this star could be still in the PMS phase.

SAO 151224 = HY CMa: an eclipsing binary (Cutispoto et al. 1995) that appears as SB2 in our spectra. The HIPPARCOS distance has a large uncertainty and thus it is difficult to assign a well defined spectral classification. Osten \& Saar (1998) inferred K3IV + K1IV spectral types. The $v \sin i$ of the secondary component derived by us $\left(11 \pm 2 \mathrm{~km} \mathrm{~s}^{-1}\right)$ is in agreement with the value reported by Osten \& Saar (1998). However, we got a rather larger value for the $v \sin i$ of the primary component and the resulting radii of $3.8 R_{\odot}$ and 1.6 $R_{\odot}$ for the primary and the secondary components, respectively, seems to us more adequate for class $\mathrm{V} / \mathrm{IV}$ than for class IV stars. We note that, although they fit the colors equally well, the tentative spectral classifications reported by Cutispoto et al. (1995) do not agree with the inferred radii.

SAO 196024: we find it to be a variable star with a photometric period of $1.422 \pm 0.007$ days (Fig. 26). The colors and the spectral signatures indicate a K0V classification. There is another possible photometric period of $2.47 \pm 0.02$ days. However, such longer period would imply a radius that is too large for a $\mathrm{K} 0$ dwarf.

SAO $234124=\mathrm{XZ}$ Pic: is an SB1 system, as shown by our RV measurements. This star is reported as an unsolved variable in the HIPPARCOS variability annex. Our photometric observations (Fig. 27) detected variability with a period of $1.87 \pm 0.02$ days. The inferred spectral classification is the one that best fits the spectral characteristics, the colors and the distance measured by HIPPARCOS.

SAO 243278: ia a component of a triple visual system. Components A+B (= HD 143474) are two bright Atype stars separated by 0.191 arcsec, whose distance has been measured by HIPPARCOS. Component C, at about 11 arcsec from HD 143474 is SAO 243278. 
It is reported to be a $\mathrm{G} 8 \mathrm{~V}+\mathrm{K} 4 \mathrm{~V}$ binary with an orbital period of 0.82376 days (Mason et al. 1995); our spectroscopic observations confirm the SB2 nature (see also Tagliaferri et al. 1999). We assume that SAO 243278 is physically linked to HD 143474. Consequentely, the HIPPARCOS distance, the spectral characteristics and the observed colors are better matched by a $\mathrm{G} 6 \mathrm{~V}+\mathrm{K} 0: \mathrm{V}$ system.

Acknowledgements. Stellar activity research at Catania Astrophysical Observatory is supported by the Italian "Ministry for Universities and Research" (MURST) and the "National Research Council" (CNR: Gruppo Nazionale di Astronomia) that are gratefully acknowledged. This research has made use of the SIMBAD database, operated at CDS, Strasbourg, France. A special thank is also due to the ESO staff for the collaboration and technical support during the observations, although the discouraging policy of ESO toward small telescopes will not make studies like the present one feasible in the future, and to Ms. Gina Santagati for revising the manuscript. Last but not least we thank the referee, Dr. M.F. Sterzik, for his usefull comments on the first version of this paper.

\section{References}

Anders G.J., Jeffries R.D., Kellett B.J., Coates D.W., 1993, MNRAS 265, 941

Andersen J., Nordstrom B., Andeberg A., et al., 1985, A\&AS 59,15

Amado P.J., Byrne P.B., 1997, A\&A 319, 967

Barstow M.A., Holberg J.B., Fleming T.A., et al., 1994, MNRAS 270, 499

Beavers W.I., Eitter J.J., 1986, ApJS 62, 147

Bessel M.S., 1990, A\&AS 83, 357

Bopp B.W., Ake T.B., Goodrich B.D., et al., 1985, ApJ 297, 691

Brandner W., Alcala J.M., Kunkel M., Moneti A., Zinnecker H., 1996, A\&A 307, 121

Burleigh M.R., Barstow M.A., Fleming T.A., 1997, MNRAS 287,381

Cayrel de Strobel G., Hauck B., Francois P., et al., 1992, A\&AS 95, 273

Craig N., Abbott M., Finley D., et al., 1997, ApJS 113, 131

Cutispoto G., 1998, A\&AS 131, 321

Cutispoto G., 1999 (in preparation)

Cutispoto G., Kürster M., Messina S., Rodonò M., Tagliaferri G., 1997a, A\&A 320, 586

Cutispoto G., Kürster M., Pagano I., Rodonò M., 1997b, IBVS 4419

Cutispoto G., Pallavicini R., Kürster M., Rodonò M., 1995, A\&A 297, 764

Cutispoto G., Tagliaferri G., Kürster M., Messina S., Rodonò M., 1998, in " The Tenth Cambridge Workshop on Cool Stars, Stellar Systems and the Sun", Donahue R.A. and Bookbinder J.A. (eds.) ASP Conf. Ser. 154, 1397

Cutispoto G., Tagliaferri G., Pallavicini R., Pasquini L., Rodonò M., 1996, A\&AS 115, 41

Duquennoy A., Mayor M., 1991, A\&A 248, 485

Favata F., Barbera M., Micela G., Sciortino S., 1993, A\&A 277,428
Favata F., Barbera M., Micela G., Sciortino S., 1995, A\&A 295,147

Fekel F.C., 1996, AJ 112, 269

Fekel F.C., 1997, PASP 109, 514

Fekel F.C., Strassmeier K.G., Weber M., Washuettl A., 1999, A\&A (in press)

Fleming T.A., Gioia I.M., Maccacaro T., 1989a, ApJ 340, 1011

Fleming T.A., Gioia I.M., Maccacaro T., 1989b, AJ 98, 692

Fleming T.A., Liebert J., Gioia I.M., Maccacaro T., 1988, ApJ 331,958

Henry G.W., Fekel F.C., Hall D.S., 1995, AJ 110, 2926

Henry T.J., Franz O.G., Wasserman L.H., et al., 1998, ApJ (in press)

Hodgkin S.T., Pye J.P., 1994, MNRAS 267, 840

Horch E.P., Ninkov Z., Slawson R.W., 1997, AJ 114, 2117

Jeffries R.D., 1995, MNRAS 273, 559

Jeffries R.D., Bertram D., Spurgeon B.R., 1995, MNRAS 276, 397

Jeffries R.D., Jewell S.T., 1993, MNRAS 264, 106

Kellett B.J., Bromage G.E., Brown A., et al., 1995, ApJ 438, 364

Landsman W., Simon T., Bergeron P., 1993, PASP 105, 841

Latham D.W., Mazeh T., Carney B.W., et al., 1988, AJ 96, 567

Mason K.O., Hassall B.J.M., Bromage G.E., et al., 1995, MNRAS 274, 1194

McGale P.A., Pye J.P., Barber C.R., Page C.G., 1995, MNRAS 275,1232

Micela G., Favata F., Sciortino S., 1997, A\&A 326, 221

Mullis C.L., Bopp B.W., 1994, PASP 106, 822

Neuhäuser R., Brandner W., 1998, A\&A 330, L29

Neuhäuser R., Torres G., Sterzik M.F., Randich S., 1997, A\&A 325,647

Osten R.A., Saar S.H., 1998, MNRAS 295, 257

Ottmann R., Fleming T.A., Pasquini L., 1997, A\&A 322, 785

Pasquini L., Lindgren H., 1994, A\&A 288, 179

Perryman M.A.C. and the HIPPARCOS Science Team, 1997, European Space Agency SP-1200, Vols. 1-12. ESA Publication Division, c/o ESTEC, Noordwijk, The Netherlands

Pounds K.A., Allan D.J., Barber C., et al., 1993, MNRAS 260, 77

Pye J.P., McGale P.A., Allan D.J., et al., 1995, MNRAS 274, 1165

Robb R.M., Gladders M.D., 1996, IBVS 4412

Rodonò M., Cutispoto G., Messina S., 1994, A\&A 281, 756

SAAO Annual Report, 1993, p. 16

Schachter J.F., Remillard R., Saar S.H., et al., 1996, ApJ 463, 747

Soderblom D.R., King J.R., Henry T.J., 1998, AJ 116, 396

Soderblom D.R., Pendleton J., Pallavicini R., 1989, AJ 97, 539

Sterzik M.F., Schmitt J.H.M.M., 1997, AJ 114, 1673

Tagliaferri G., Cutispoto G., Pallavicini R., Randich S., Pasquini L., 1994, A\&A 285, 272

Tagliaferri G., Cutispoto G., Pastori L., et al., 1999 (in preparation)

Tagliaferri G., Randich S., Pallavicini R., Cutispoto G., 1992, in "High Resolution Spectroscopy with the VLT", ESO Proceedings, Ulrich M.-H. (ed.). Garching, Germany, p. 171

Vennes S., Christian D.J., Thorstensen J.R., 1998, ApJ 502, 763

Weis E.W., 1991, AJ 102, 1795 